\title{
ON THE PROBLEM OF THE INVARIANCE OF HOMO- TOPICAL STABILITY OF POINTS UNDER CARTESIAN MULTIPLICATION
}

\section{HIROSHI NOGUCHI}

1. Introduction. The principal purpose of this paper is to give a negative solution for the problem of Borsuk and Jaworowski [3, p. 164 ], ${ }^{1}$ that is to say, in $\$ 4$ we prove that Begle's example [2] shows that the homotopical stability of points is not invariant under Cartesian multiplication (Theorem 4).

In $\$ 2$ we investigate set-theoretic properties of the joins of spaces. The relation between our problem and the join is also described in $\$ 2$ (Theorem 1). In $\$ 3$ homology groups and fundamental groups of the joins are considered.

2. Set-theoretic properties of the joins. Throughout the paper all spaces are finite complexes (=finite polyhedra) and nonvacuous, unless the contrary is explicitly stated. The equality between two spaces implies they are homeomorphic.

Definition 1. Let $A$ and $B$ be spaces, their join $A * B$ is the space obtained from $A \times B \times I$ by identifying each set of the form $a \times B \times 1$ with $a \in A$ and each set of the form $A \times b \times 0$ with $b \in B$, where $I$ denotes the interval $0 \leqq t \leqq 1$. Let either $A$ or $B$, say $B$, be vacuous, their join is $A$ itself. Let either $A$ or $B$, say $B$, be a point $b$, their join will be called a cone $\widehat{A}$ of the base $A$ and the vertex $b$. Let either $A$ or $B$, say $B$, be the $(n-1)$-sphere $S^{n-1}(n \geqq 1)$, their join will be called an $n$-fold suspension of $A$.

Under the identification $\omega_{A * B}, A$ and $B$ may be regarded as subsets $\omega_{A * B}(A \times B \times 1)$ and $\omega_{A * B}(A \times B \times 0)$ of $A * B$ respectively.

Lemma 1. (i) $A * B=B * A$,

(ii) if $A=\bigcup_{i=1}^{n} A_{i}, A * B=\bigcup_{i=1}^{n}\left(A_{i} * B\right)$,

(iii) if $A=\bigcap_{i=1}^{n} A_{i}, A * B=\bigcap_{i=1}^{n}\left(A_{i} * B\right)$, where $n$ is a positive integer.

Proof. (i) Let $h: A \times B \times I \rightarrow B \times A \times I$ be a homeomorphism defined by taking $h(a, b, t)=(b, a, 1-t)$ and let $\psi \equiv \omega_{B * A} h \omega_{A * B}^{-1}$. It is easily checked that $\psi$ is a $1: 1$-transformation. From Lemma 3 of $[8], \psi$ is a homeomorphism. (ii) Since $\omega_{A * B} \mid A_{i} \times B \times I=\omega_{A_{i} * B}$, it follows that

Received by the editors May 6, 1954 and, in revised form, October 18, 1954.

1 Numbers in brackets refer to the bibliography at the end of the paper. 
$A * B \supset \bigcup_{i=1}^{n}\left(A_{i} * B\right)$. For each point $x \in A * B$ we may find $a \in A_{i}$ such that $x=\omega_{A_{i} *}(a, b, t)$. Hence we have $A * B \subset \bigcup_{i-1}^{n}\left(A_{i} * B\right)$. (iii) It is similar to (ii).

Lemma 2. $A * B$ is arcwise-connected.

Proof. If both $A$ and $B$ are arcwise-connected, from Definition 1 $A * B$ is arcwise-connected. In the general case, let $x$ and $y$ be arbitrary points of $A * B$. From Lemma 1 there are arcwise-connected components $A^{\prime}, A^{\prime \prime}$ of $A$ and $B^{\prime}, B^{\prime \prime}$ of $B$ such that $x \in A^{\prime} * B^{\prime}$, $y \in A^{\prime \prime} * B^{\prime \prime}$ and $A^{\prime} * B^{\prime} \cap A^{\prime \prime} * B^{\prime} \supset B^{\prime}, A^{\prime \prime} * B^{\prime} \cap A^{\prime \prime} * B^{\prime \prime} \supset A^{\prime \prime}$. It follows that $x$ and $y$ are in an arcwise-connected component of $A * B$. This completes the proof.

\section{Lemma 3. $\hat{A} \times \widehat{B}=(A \times \widehat{B} \cup \hat{A} \times B)$.}

Proof. Let $\widehat{A}=A * a_{0}, \widehat{B}=B * b_{0}$ and $(A \times \widehat{B} \cup \widehat{A} \times B)^{-}=(A \times \widehat{B}$ $\cup \widehat{A} \times B) * c$. From Lemma 4 of [8], $\omega_{\widehat{A} \times \widehat{B}} \equiv\left(\omega_{\widehat{A}}, \omega_{\widehat{B}}\right), \omega_{A \times \widehat{B}} \equiv$ (identity, $\left.\omega_{\widehat{B}}\right)$ and $\omega_{\widehat{A} \times B} \equiv\left(\omega_{\widehat{A}}\right.$, identity $)$ are identifications. Let $P$ be a space obtained from $A \times B \times b_{0} \times I \cup A \times a_{0} \times I \times B$ by identifying each point $\left(a, b, b_{0}, 1\right)$ with $\left(a, a_{0}, 1, b\right)$ and $\omega_{1}$ be the identification. Then from Lemma 3 of [8] we have an identification $\omega_{2}: P \rightarrow A \times \widehat{B} \cup \hat{A} \times B$ such that $\omega_{2} \omega_{1}\left|A \times B \times b_{0} \times I=\omega_{A \times \widehat{B}}, \omega_{2} \omega_{1}\right| A \times a_{0} \times I \times B=\omega_{\widehat{A} \times B}$. Let $\omega_{3}$ be the identification $\omega_{3}:(A \times \widehat{B} \cup \widehat{A} \times B) \times c \times I \rightarrow(A \times \widehat{B} \cup \widehat{A} \times B)$ - . Let $f: A \times a_{0} \times I \times B \times b_{0} \times I \rightarrow(A \times \widehat{B} \cup \hat{A} \times B)^{\wedge}$ be a transformation defined by taking

$$
f\left(a, a_{0}, t, b, b_{0}, t^{\prime}\right)= \begin{cases}\omega_{3}\left(\omega_{2} \omega_{1}\left(a, b, b_{0}, t^{\prime} / t\right),(c, t)\right) & \text { for } t \geqq t^{\prime}, \\ \omega_{3}\left(\omega_{2} \omega_{1}\left(a, a_{0}, t / t^{\prime}, b\right),\left(c, t^{\prime}\right)\right) & \text { for } t^{\prime} \geqq t,\end{cases}
$$

where $0 / 0$ means 1 .

Let $\phi \equiv f \omega_{\widehat{A} \times \widehat{B}}^{-1}: \widehat{A} \times \widehat{B} \rightarrow(A \times \widehat{B} \cup \widehat{A} \times B)$ - . It is easily checked that $f$ is a map and $\phi$ is a $1: 1$-transformation. Therefore the lemma follows from Lemma 3 of [8].

Lemma 4. $A \times \widehat{B} \cup \widehat{A} \times B=A * B$.

Proof. Let $g: A \times B \times b_{0} \times I \cup A \times a_{0} \times I \times B \rightarrow A \times B \times I$ be a map defined by taking

$$
\begin{aligned}
& \left(g \mid\left(A \times B \times b_{0} \times I\right)\right)\left(a, b, b_{0}, t\right)=(a, b, 1-t / 2), \\
& \left(g \mid\left(A \times a_{0} \times I \times B\right)\right)\left(a, a_{0}, t, b\right)=(a, b, t / 2),
\end{aligned}
$$

and let $\kappa \equiv \omega_{A * B} g \omega_{1}^{-1} \omega_{2}^{-1}: A \times \widehat{B} \cup \widehat{A} \times B \rightarrow A * B$, it is easily checked that $\kappa$ is a $1: 1$-transformation. From Lemma 3 of [8] $\kappa$ is a homeomorphism. 
TheOREM 1. Let $K, L$ be complexes and $a$, a vertex of $K, A$ a neighborhood complex of $a$ and $b, a$ vertex of $L, B$ a neighborhood complex of $b$. Then $A * B$ is a neighborhood complex of $(a, b)$ in $K \times L$.

Proof. From Lemma 3 and the definition of the neighborhood complex [6, $\S 3], A \times \hat{B} \cup \widehat{A} \times B$ is a neighborhood complex of $(a, b)$ in $K \times L$. Therefore the theorem follows from Lemma 4 .

\section{Homology groups and fundamental groups of the joins.}

Definition 2. Let $A, B$ be complexes augmented by the nullsimplex. The join $A * B$ of $A$ and $B$ is the augmented complex whose vertices form the union of the vertices of $A$ and $B$ respectively. A set of vertices are those of a simplex if the join of its subsets in $A$ and $B$ span a simplex there [5, p. 137].

From Lemma 1 it readily follows that Definition 2 and Definition 1 are equivalent.

We already know the boundary relation for the join;

$$
F\left(\xi^{p} * \eta^{q}\right)=\left(F \xi^{p}\right) * \eta^{q}+(-1)^{p+1} \xi^{p} *\left(F \eta^{q}\right),
$$

where $\xi^{p}$ is a $p$-dimensional chain of $A, \eta^{q}$ is a $q$-dimensional chain of $B$, and $F$ denotes the boundary operation [5, p. 139].

Let $H_{r}(A)$ and $T_{r}(A), r \geqq 1$, be the integral $r$-dimensional homology group of $A$ and the integral $r$-dimensional torsion group of $A$ respectively and $H_{0}(A)$ be the reduced (berandungsfähigen) 0 -dimensional homology group of $A[1$, p. 209].

Then by Lefschetz's method [5, p. 137] and Künneth's theorem $[1$, p. 308] we can deduce;

Theorem 2. $H_{n}(A * B) \approx H_{n-1}(A \times B) \approx \sum_{p+q=n-1}\left(H_{p}(A) \otimes H_{q}(B)\right)$ $\oplus \sum_{r+s=n-2}\left(T_{r}(A) \otimes T_{s}(B)\right)$ for $n \geqq 0$, where the symbols $\sum$ and $\oplus$ signify the direct sum of groups and $\otimes$ signifies the tensor product of groups.

CoROLlaRy. The homology groups of the $n$-fold suspension of $A$ are

$$
\begin{array}{lrl}
H_{p}\left(A * S^{n-1}\right) \approx H_{p-n}(A) \otimes H_{n-1}\left(S^{n-1}\right) \approx H_{p-n}(A) & \text { for } p \geqq n, \\
H_{p}\left(A * S^{n-1}\right) \approx 0 & \text { for } 0 \leqq p<n .
\end{array}
$$

THEOREM 3. Let either $A$ or $B$ be arcwise-connected, then the fundamental group of the join of $A$ and $B$ is trivial, that is to say, $\pi_{1}(A * B)$ $\approx$ unity.

Proof. In the first place we assume that both $A$ and $B$ are arcwiseconnected. From Lemma 2 and the assumption complexes $A \times \widehat{B}$, $\hat{A} \times B$, and $A \times B$ are arcwise-connected. From Theorem 1 of $[7, \mathrm{p}$. 
177] and Lemma $4, \pi_{1}(A * B)=\pi_{1}(\kappa(A \times \widehat{B} \cup \widehat{A} \times B))$ is a factor group of the free product $\pi_{1}(\kappa(A \times \widehat{B})) \bigcirc \pi_{1}(\kappa(\widehat{A} \times B))$. Since $\pi_{1}(A \times \widehat{B})$ $\approx \pi_{1}(A \times b)$ and $\pi_{1}(\widehat{A} \times B) \approx \pi_{1}(a \times B), \pi_{1}(A * B)$ is a factor group of $\pi_{1}(\kappa(A \times b)) \bigcirc \pi_{1}(\kappa(a \times B))$, where $a, b$ are fixed points of $A, B$ respectively. It is easily seen that each closed path of $\kappa(A \times b)$ which passes $\kappa(a, b)$ and $\kappa(a, b)$ are homotopic in the cone $\omega_{A{ }^{*} B}(A \times b \times I)$ relative to $\kappa(a, b)$. Since each generator of $\pi_{1}(\kappa(A \times b))$ is null-homotopic in $A * B$ and the same is also true for $\pi_{1}(\kappa(a \times B))$, then $\pi_{1}(A * B) \approx$ unity.

In the next place we assume that either $A$ or $B$, say $A$, is arcwiseconnected. Let $B_{1}, \cdots, B_{n}$ be arcwise-connected components of $B$, from Lemma $1, A * B=A * B_{1} \cup \ldots \cup A * B_{n}$ and $A * B_{i} \cap A * B_{j}=A$, where $i \neq j$ and $1 \leqq i, j \leqq n$. Since $A * B_{1}, \cdots, A * B_{n}$ are arcwiseconnected, $\pi_{1}(A * B)$ is a factor group of the free product $\pi_{1}\left(A * B_{1}\right)$ $\bigcirc \cdots \bigcirc \pi_{1}\left(A * B_{n}\right)$. Thus the theorem follows from the first consideration.

Corollary. $A * B$ is noncontractible if, and only if, there exists $p \geqq 0$ such that $H_{p}(A \times B)$ not $\approx 0$.

Proof. Necessity: If $A * B$ is noncontractible, either $\pi_{1}(A * B)$ not $\approx$ unity or $H_{n}(A * B)$ not $\approx 0, n \geqq 2$. If $\pi_{1}(A * B)$ not $\approx$ unity, from Theorem 3 neither $A$ nor $B$ is arcwise-connected. Then we have $H_{0}(A \times B)$ not $\approx 0$. If $H_{n}(A * B)$ not $\approx 0, n \geqq 2$, from Theorem 2 , $H_{n-1}(A \times B)$ not $\approx 0$. Sufficiency: From Theorem 2 it is trivial.

REMARK. From the above facts we can easily deduce:

Let either $A$ or $B$ be arcwise-connected and $H_{i}(A) \approx 0, H_{j}(B) \approx 0$ for $i<p, j<q$. Then $\pi_{p+q+1}(A * B) \approx H_{p}(A) \otimes H_{q}(B)$, where $\pi_{r}(A)$ denotes $r$-dimensional homotopy group of $A, r \geqq 2$.

\section{Application to the homotopical stability of points.}

Example. Let $A$ be a complex which is obtained from a Poincaré's sphere with nonvanishing fundamental group by omitting an open 3 -simplex and let $K=\left(a_{0} \cup a_{1}\right) * A$ and let $L=\left(b_{0} \cup b_{1}\right) * S^{n-1}$. Then from Theorem $1, A * S^{n-1}$ is a neighborhood complex of $\left(a_{0}, b_{0}\right)$ in $K \times L$. From Theorems 3.1 and 3.2 of [6] and corollaries of Theorems 2 and 3 , we have that $a_{0}$ and $b_{0}$ are homotopically stabile but $\left(a_{0}, b_{0}\right)$ is homotopically labile. When $S^{n-1}=S^{0}$, then the example is the one of Begle [2].

Thus we have;

THEOREM 4. The homotopical stability of points is not invariant under Cartesian multiplication.

From Theorem 3.2 of [6] and corollary of Theorem 3 we have: 
Theorem 5. $A$ point $(a, b)$ of $K^{\wedge} L$ is homotopically stabile if, and only if, there exist neighborhood complexes $A$ of $a$ in $K$ and $B$ of $b$ in $L$ such that $H_{p}(A \times B)$ not $\approx 0$ for some $p \geqq 0$.

REMARK. We may propose an analogous problem for the stability of points owing to Hopf and Pannwitz [4]. From Theorem 6 of [4], the boundary relation for the join in $\$ 3$ and Theorem 1, we can deduce;

For homogeneous complexes the stability of points owing to Hopf and Pannwitz is invariant under Cartesian multiplication.

\section{BIBLIOGRAPHY}

1. P. Alexandroff and H. Hopf, Topologie I, Berlin, 1935.

2. E. G. Begle, Intersections of contractible polyhedra, Bull. Amer. Math. Soc. vol. 49 (1943) pp. 386-387.

3. K. Borsuk and J. W. Jaworowski, On labil and stabil points, Fund. Math. vol. 39 (1952) pp. 159-175.

4. H. Hopf and E. Pannwitz, Über stetige Deformationen von Komplexen in sich, Math. Ann. vol. 108 (1933) pp. 433-465.

5. S. Lefschetz, Algebraic topology, New York, 1942.

6. H. Noguchi, A characterization of homotopically labil points, Kodai Math. Semi. Rep. vol. 6 (1954) pp. 13-16.

7. H. Seifert and W. Threlfall, Lehrbuch der Topologie, Berlin, 1934.

8. J. H. C. Whitehead, Note on a theorem due to Borsuk, Bull. Amer. Math. Soc. vol. 54 (1948) pp. 1125-1132.

WASEDA UNIVERSITY, TOKYo 